Voix et Images

\title{
Gilles Marcotte ou la pensée critique de l'inachèvement
}

Joseph Bonenfant

Volume 6, numéro 1, automne 1980

Gilles Marcotte

URI : https://id.erudit.org/iderudit/200249ar

DOI : https://doi.org/10.7202/200249ar

Aller au sommaire du numéro

Éditeur(s)

Les Presses de l'Université du Québec

ISSN

0318-9201 (imprimé)

1705-933X (numérique)

Découvrir la revue

Citer cet article

Bonenfant, J. (1980). Gilles Marcotte ou la pensée critique de l'inachèvement.

Voix et Images, 6(1), 51-61. https://doi.org/10.7202/200249ar d'utilisation que vous pouvez consulter en ligne.

https://apropos.erudit.org/fr/usagers/politique-dutilisation/ 


\title{
Gilles Marcotte ou la pensée critique de l'inachèvement
}

\author{
par Joseph Bonenfant
}

C'est l'ignorance, aujourd'hui, qui est difficile. Le savoir est donné, imposé; c'est l'ignorance qui doit être gagnée.

M. G., Liberté, no, 84, 1972, p. 112.

La critique aussi est un discours de fiction. Elle peut moins que la poésie, ou le roman, s'abstenir d'idées, ou se retenir de refléter l'humeur de son auteur, ou cacher sa science. Mais là n'est pas l'important. J'aurai l'occasion de signaler, chez Gilles Marcotte, les idées, avec leur constance et leur force, d'évoquer son attitude de lecteur et sa vaste culture. Comme ces choses-là pourront être claires! II y a risque qu'elles dessinent les configurations d'un savoir qui serait bien l'image la plus fausse qui se dégage de cette critique. Là, pas d'arcane, pas de recette, ni de théorie lourde. Plutôt une œuvre de fiction, mettant en action mille personnages vivants: les écrivains, les travailleurs littéraires et leurs mondes de papier. Comme toute critique, la sienne est une prospection du monde au deuxième degré, une exploration incertaine et, malgré les apparences, un des plus fascinants domaines du non-savoir. Jamais cette critique ne souffre (ni ne jouit de la «crampe » théorique.

\section{Le plaisir}

Partons de la notion de plaisir, de ce qui ne donne pas lieu à une science. Elle est à la base de tous ses écrits; elle ne se cache pas. Comme elle n'est pas sur-utilisée, on ne peut y voir une excuse ou un paravent. "Je pense aussi qu'un livre de critique doit être de la littérature. S'il n'y a pas de plaisir d'écriture pour le critique, ça m'intéresse moins "1. Voilà pour la déclaration de principe. La même notion barthienne se retrouve dans la présentation des Bonnes rencontres: "J'espère seulement qu'on y trouvera le reflet du plaisir que j'ai eu, pendant des années, à découvrir des livres nouveaux, à les interroger, à les commenter "2. Cette attitude désarmante, nullement désarmée, devient un principe de lecture 
quand elle projette son idée de plaisir sur l'objet même à étudier. Les écrivains analysés dans le Roman à l'imparfait sont gratifiés de cette faculté de jouir de l'écriture: "Le souci de bien écrire leur est étranger: ils écrivent, tout simplement, à toute volée, avec un impudent plaisir " ${ }^{3}$. Au-delà de la "parlure secrétariale" chez Godbout, de l'absence d'un "style continu " chez Marie-Claire Blais, des "jeux de la rhétorique et du vocabulaire " chez Bessette, d'un "monde linguistique parfaitement approprié " chez Ducharme, ce qui est lu et mis en lumière, c'est un maniement jouissif de la langue, reconnue comme étant "leur famille, leur corps, leur pays" 4 .

Une autre attitude du critique, celle-là plus caractéristique, est l'affirmation visible, insistante de ce que j'appellerais la modestie critique. C'est une absence de prétention qui, en se nommant, joue le rôle phatique d'établir une bonne communication, mais surtout qui signale une conscience des limites. Au début du Temps des poètes, le critique avertit: "Qu'une telle sympathie, vouée aux cuvres plutôt qu'aux systèmes, suscite une démarche parfois peu ordonnée, cela va de soi; je préfère ce désordre à une formalisation prématurée. Avant de définir précisément la topographie d'un domaine, il faut l'explorer. C'est, modestement, ce que j'ai tenté de faire " 5 . La même idée revient, sous forme négative, au début des Bonnes rencontres: "Ce petit livre, qui n'a évidemment aucune prétention à la synthèse, dans quelque domaine que ce soit" 6 . Ailleurs, l'attitude est là, mais non conceptualisée: «Je livre des matériaux qui, je l'espère, ne sont pas sans utilité"? ${ }^{7}$ Ou encore, au début du Roman à l'imparfait: "Mon but n'était d'ailleurs pas d'écrire des études exhaustives (...) mais d'ouvrir quelques voies de réflexion " ${ }^{8}$. L'avantage de ces déclarations liminaires est de relativiser une position critique qui préfère toujours mettre l'œuvre en relation avec un lecteur (celui qui a lu et celui qui lira) plutôt que de la mettre à l'épreuve d'une position théorique. II y a comme un malin plaisir ici à mettre de l'avant cette modestie stratégique. Lecteur, de quoi seras-tu déçu si tu ne t'attends pas à autre chose qu'à ces simples notes? Serais-tu déçu par un autre que toi-même? Cette relativisation consiste toujours à s'impliquer comme locuteur plutôt que comme théoricien. A parler comme sujet plutôt qu'à faire parler la théorie. Ainsi lit-on, au cœur d'un article: "Cette interprétation du texte de Lautréamont est aléatoire, de même que la relation dans laquelle je le fais entrer avec la pensée de Bataille " ${ }^{9}$. Je trouve bon que l'absence de dogmatisme s'énonce clairement et que la conscience du plaisir s'accroisse de celle de la modestie. Voyez, je vous cacherai plus ma modestie que mon plaisir, veuillez m'en savoir gré.

Marcotte excelle, en d'autres manières, à prendre de la distance: «ll n'y a pas là de quoi fouetter un chat ${ }^{10}$; “après avoir souri avec indulgence, nous constaterons que..." 11 . Cette facilité du repli est une forme d'humour. Seul un critique qui ne se prend pas au sérieux peut faire de telles cabrioles. "Mais cette métaphore a assez filé. Elle est en train de devenir une allégorie. Passons aux choses sérieuses "12. À cette facilité insolente qui se joue de la transition, on pourrait ajouter des traits humoristiques 
dans le genre de ceci: “Quelques autres pétards ont éclaté ici et là. Et comme, au Québec, on confond souvent pétards et bombes atomiques, on a parfois conclu à la fin du monde "13; ou encore une flèche satirique: “L'avant-garde, au Québec, ça nous connaît. Dans l'action culturelle, comme dans l'enseignement des mathématiques ou celui du français, nous courons plus vite que tout le monde, nous avons déjà un pied dans le vingt et unième siècle "14. Il va de soi que l'humour ou la satire sont des marques d'absence de prétention; ce sont les preuves rhétoriques du plaisir. II est certain que l'un et l'autre pourraient être pris en mauvaise part et être stigmatisés par quelqu'un qui ne jurerait que par les théories. Mais Marcotte a prévu le coup. Non seulement il ne se veut pas théoricien mais nous verrons dans quelle mesure cette humilité contredit la réalité mais encore tient-il à dire qu'il est un critique issu du journalisme: «Je suis parti d'une expérience principalement de journaliste sur quoi j'ai beaucoup compté "15.

\section{Le journalisme}

S'il fallait à tout prix, à la manière de Georges Poulet, formuler le cogito de cette pensée critique, on partirait de la motivation pressante qui fait lire et écrire le journaliste impénitent qu'il est. Ce serait quelque chose comme: je lis, j'informe, donc j'écris. Car, ne l'oublions pas, Marcotte est avant tout un communicateur littéraire qui doit le meilleur de sa formation aux dures exigences du journalisme. Ce ne sont pas de longues études doctorales qui l'ont formé, ni une recherche de type universitaire et bénédictin qui lui ont donné ses galons dans la critique: c'est le souci de faire parler les cuvres et par leur truchement de parler au plus vaste auditoire possible. Il lui a toujours plus importé de se commettre dans sa critique, même au gré de l'incertitude et du tâtonnement, que d'y importer et d'y exploiter des concepts (critiques ou théoriques) venus d'ailleurs. Quel dommage, quel cirque aurait-ce été si, depuis trente ans qu'il signe des études critiques, notre auteur avait suivi toutes les modes qui se présentaient: l'existentialisme sartrien, l'objectalisme du nouveau roman, le sociologisme de Lukacs, le déterminisme de Goldmann, le thématisme de Richard, le catégoricisme de Poulet, les diverses sémiologies, poétiques et pragmatiques. Rien de tout cela, certes, ne lui est étranger, mais aucune de ces approches ne lui sert de planche de salut, de point de départ exclusif. Pas de palliatif méthodique, plutôt la méthode journalistique.

G. Marcotte sait bien que la littérature répond toujours quelque chose au sociologue, au psychanalyste, au linguiste qui l'interroge. Mais au journaliste, que peut-elle apporter? Impossible de mettre tous ces praticiens sur le même pied. Le journalisme, relevant plus des sciences sociales que des sciences humaines, exige un sens de l'observation du champ culturel et un sens de la communication du texte littéraire incomparablement plus aigus. Sous ce rapport, la position théorique la plus constante 
ici est celle qui fonde, non tant le rapport de la société à la littérature, que celui du texte littéraire au texte sociologique. De même que pour le sociologue la société est “société lue, devenue texte» ${ }^{16}$, ainsi en est-il de la littérature pour le critique. La littérature reprend, module, transforme la société en s'écrivant, de même que le critique, en produisant sa lecture, transforme la littérature en la modulant, en la modalisant selon des perspectives variées. Cette transformation des champs (social, culturel, littéraire, critique) me semble être le postulat de base de sa critique, même si des effets de lecture peuvent parfois permettre de penser le contraire, comme lorsque Jean Éthier-Blais, parlant du Temps des poètes, écrit: «L'analyse de M. Gilles Marcotte est plus thématique qu'historique. Je trouve que I'histoire est un peu trop absente de son livre „17. Tel livre, tel article peuvent donner cette impression, mais je suis convaincu, après avoir parcouru l'ensemble de l'cuvre critique, que cette affirmation ne vaut pas pour tout le reste. C'est sans doute tardivement que cette pensée critique a pris conscience de notre société et de son histoire, tardivement aussi qu'elle a pris conscience de l'importance du langage dans l'expérience littéraire. Mais ces dimensions - societé et langage - sont désormais acquises et actives, omniprésentes dans sa critique la plus actuelle. Bref, la littérature est pour lui le langage qu'une société s'adresse à ellemême pour susciter, accroître et évaluer ses propres transformations. Tout est dans cette représentation.

\section{La méthode en question}

II ne s'est jamais embarrassé d'un appareillage méthodologique. II cite à l'occasion les penseurs importants du siècle, soit pour les prendre à rebours, soit pour se couvrir de leur autorité, soit encore pour approfondir les perspectives. La conséquence en est que cette critique n'a pas tendance à se perdre dans des divagations idéologiques, pour le plaisir même de la chose. Ce discours critique s'amarre à l'œuvre analysée; le texte lisant reste étroitement tributaire du texte lu. La lecture reste consciente d'elle-même, de sa coordination, et même de sa subordination à l'objet primordial. La pratique critique est tellement assurée qu'elle peut oser sans risque s'attaquer à l'esprit de système qui cherche à pénétrer la critique littéraire. "On s'affole beaucoup dans les universités, ces temps-ci " écrit-il en 1974. "Les théories se succèdent à un rythme étourdissant, les professeurs ne savent plus où donner de la tête et il se trouve des étudiants pour demander qu'on fasse, enfin, le tour des «méthodes» offertes à l'étalage pour en choisir la plus solide, la plus sûre"18. On n'imagine pas qu'un jeune professeur, frais émoulu de son doctorat, tienne de tels propos. L'assurance se fonde sur une longue tradition de la critique dite créatrice. Quelle est la méthode d'un Thibaudet, d'un Blanchot, d'un Ethier-Blais? Rien d'autre qu'un style bien à soi. Quand Robert Vigneault signale chez G. Marcotte "la pensée vigoureuse", la «langue remarquablement ferme", la «vaste culture littéraire», «l'indiscutable bonheur d'expression», "l'am- 
pleur de vue», “les intuitions pénétrantes», et qu'il ajoute: “Le mérite particulier de G.M. est d'allier assez souvent le caractère expéditif de la chronique et le jugement sûr du critique réfléchi "19, même si ces remarques s'appliquent aux Bonnes rencontres, force nous est de dire, outre de faire la bonne mesure d'éloges, qu'elles valent aussi pour le reste de l'œuvre critique. La sûreté du jug ement ne tient pas lieu de méthode; elle l'assume et la transcende; elle relève plus d'une valeur que d'un sens et son rapport à l'intelligence n'est pas moindre que son rapport à l'émotion. Mais quand $R$. Vigneault le qualifie de "critique aimablement conservateur", et "d'humaniste imperturbable», on peut être moins d'accord. L'humanisme est un bien grand mot et je ne le trouve grave que s'il est idéaliste. Or G. Marcotte ne croit-il pas à la matérialité du texte; ne peut-on parler dans son cas d'un matérialisme descriptif, analytique? Pour ce qui est du conservateur, il faudrait voir comment son opposé, l'idéologue, pratique la critique et pousse son progressisme. L'œuvre ne devient-elle pas alors matière théorisable et pièce à conviction pour instruire des procès?

Le problème est le suivant: l'œuvre littéraire a censément une autonomie, et en même temps elle est tributaire d'un texte social. C'est le problème du référent. Que se passe-t-il dans les œuvres qui traversent les siècles? Reprenant une idée d'Escarpit, Marcotte évoque les œuvres qui «peuvent être lues sans référence, ou presque, à l'ensemble culturel qui les a vues naître". Seules ces couvres "peuvent être considérées comme littéraires ${ }^{20}$. D'autre part, les référents linguistiques et sociaux sont ce qui font comprendre l'œuvre. Cette dernière est à la fois historique dans son origine et sa formation, et trans-historique dans son sens et sa durée. Une des idées maîtresses de la critique de G. M. est que l'œuvre ne doit pas s'enfermer dans l'historique. La littérature nationale n'est pas un absolu; l'histoire du pays peut et doit transformer l'œuvre, mais ne peut être le fondement, le critère, l'aune unique de référence. C'est ce qui se passe au Québec quand on juge de toute œuvre par rapport au thème du pays ou de la révolution. L'œuvre est dans et hors de l'histoire, comme l'histoire est dans et hors de l'œuvre. II me semble que cette dimension - et cette limitation - du référent social est toujours présente dans cette pensée critique autant comme freinage que comme accélération, comme source d'adhésion que comme motif de réticence. Si l'humanisme est un individualisme, ce n'est pas celui-là qu'on trouve chez G. M. critique.

Souvent chez lui les intuitions théoriques jaillisent à travers les jugements critiques, comme des idées surgissent au cours d'un dialogue. Prenons par exemple un texte de 1977: “La révolution de la tranquillité " ${ }^{21}$, pour voir comment la conscience du social est une conscience de la transformation, soit douce soit violente. II signale d'abord que les premiers poètes de l'Hexagone ont repris les mots de leurs aînés. C'était déjà une originalité que de s'inscrire dans une continuité. «lis pillent, donc, ils imitent. Mais piller, imiter, c'est également transformer (...). Que peuvent nous dire ces transformations "? Derrière ce jugement critique, on recon- 
naît la théorie de la citation comme appropriation dans la rupture ou la parodie, l'exclusion ou le renforcement, comme allait la présenter plus tard Antoine Compagnon dans la Seconde main, le travail de la citation. G. Marcotte se plaît ici à comparer, à confronter - ces rapprochements critiques sont une technique d'analyse constante à travers toute l'œuvre Jean-Guy Pilon et Alain Grandbois. Ainsi fait-il mieux voir "l'évolution de l'écriture poétique au Québec". II confronte Gatien Lapointe et Nelligan, Luc Perrier et Saint-Denys Garneau. Non pour humilier des contemporains, mais pour démontrer qu'on n'a pas su lire encore Nelligan, Saint-Denys Garneau et Grandbois dans ce que leur poésie a de moderne, «dans toute la violence de leur déconstruction $»$. Le critique dégage certaines continuités thématiques entre jeunes et anciens poètes, mais ce traitement critique va moins loin que les considérations formelles qui dégagent la modernité de la poésie québécoise des années cinquante. Ce qui se passe ici de plus important, c'est ce «travail obscur " de la poésie en vue de sa "légitimité sociale". Il y a transformation dans le passage des aînés aux poètes de l'Hexagone, continuité substantielle mais écarts formels et visées linguistiquement différentes. Et survient à la fin une synthèse, sous forme d'allégorie, où la rupture violente est une exigence théorique appelée par ce qui précédait: "Qu'aujourd'hui, le groupe des Herbes rouges secoue le cocotier de l'Hexagone, cela se comprend comme une nécessité. Les poètes dont j'ai parlé n'avaient pas, eux, de cocotier à secouer; ils devaient le faire pousser, lui donner le temps de pousser - pour le plus grand profit des futures avant-gardes".

Rien dans ce texte ne regimbe contre la nécessité de la destructionnégation des lourdes choses passées, toutes parfaites qu'elles soient. S'il ne fait pas l'apologie systématique de la rupture idéologique, notre auteur sait au moins entrer dans la reconnaissance des transformations, lentes ou rapides. De sorte qu'on pourrait dire que la critique est toujours en retard d'une lecture sur l'œuvre, qu'elle est à sa remorque, mais aussi qu'elle amplifie les messages en même temps qu'elle les capte et les fait passer, sur son mode, désormais majeur, de l'aire individuelle au champ collectif. Ce concept de transformation s'applique ici autant à la littérature qu'à la société, non parallèlement, mais symbiotiquement. Nulle critique plus que la sienne n'est consciente de ce travail de l'histoire sur elle-même, de la société, de la littérature, de l'individu sur soi-même, et en même temps dans un système inéluctable d'interactions.

\section{Le travail de l'inachevé}

Les titres des livres ne sont-ils pas éloquents sous ce rapport? Le premier livre, en 1962, s'intitule Une littérature qui se fait. La fortune de ce titre n'est plus à démontrer, non comme trouvaille; mais comme concept opératoire de l'analyse. On trouve la même figure de l'inachèvement temporel dans le Roman à l'imparfait. Ce qui n'est pas achevé est forcément 
en voie de s'accomplir. La pensée du critique affectionne ces points intermédiaires entre un point de départ et un point d'arrivée. Cet espace intercalaire est aussi un Temps des poètes, un temps parmi d'autres, mais insistant sur la possibilité d'un accomplissement du temps et incluant aussi un temps des Bonnes rencontres. Nous n'avons pas encore de grands romans de la maturité, souligne-t-il dans le Roman à l'imparfait; c'est dire que l'achèvement n'est pas atteint; mais un déjà-là compense pour la déception d'un pas-encore. C'est jusque dans le détail de l'analyse du texte romanesque que le critique décèle l'inachèvement, quand il compare la naissance d'Emmanuel chez M.-C. Blais et celle du petit d'Alphonsine dans Trente Arpents 22: "une image globale qui restera toujours inachevée». II n'est pas jusqu'à la mobilité des formes littéraires qui ne s'explique par un inachèvement radical, de même que l'interrogation sociale, toujours en instance de perfectionnement: "Ce que raconte le roman, et la forme dans laquelle il le raconte - qui sont une même chose, puisque la forme détermine la substance du récit - sont eux-mêmes justiciables d'une interrogation sociale. Les formes littéraires (...) sont mobiles, vivantes, en relation continue avec les formes sociales 23 . C'est pourquoi, analysant le Roman à l'imparfait, qui est, avec une Litterature qui se fait, le plus beau fleuron de son œuvre critique, Jacques Michon a pleinement raison de faire de la transformation le fondement théorique de ce discours critique: "L'auteur définit toujours le texte romanesque comme un texte qui transforme d'autres textes, soit le récit réaliste chez $B \in$ isette, l'épopée chez Ducharme, le roman du terroir chez Marie-Claire Blais et le journal chez Godbout 24 .

On peut penser également que dans la mesure où son œuvre critique est elle-même inachevée, elle ira de plus en plus vers son total accomplissement en préservant et en approfondissant le discours de cette relation sans cesse inachevée entre les formes sociales et les formes littéraires, entre les formes du contenu des œuvres et les substances - linguistique, idéologique, sociologique - de ces formes, cette relation toujours mouvante entre l'œuvre et son lecteur, entre les personnages romanesques d'autrefois et de maintenant, entre les types - rhétorique, thématique, symbolique - de discours poétiques, finalement la relation toujours vivante entre les écrivains eux-mêmes. L'approche méthodique la plu constante de G. Marcotte est la pratique de l'analyse comparative. Que cette comparaison se manifeste en termes d'identité ou d'opposition, de ressemblance ou de différence, toujours elle sert à faire ressortir relations et relativisations; c'est le moyen que prend dans cette critique la visée théorique pour faire voir et rendre actives les multiples transformations que tout produit littéraire implique, à défaut d'en être conscient. C'est bien souvent le critique qui met le doigt sur une dimension qui avait échappé à l'auteur du texte. La tâche du critique n'est-elle pas de faire la synthèse idéologique de son époque à partir des œuvres de la littérature? À la fin d'une étude consacrée à Ducharme, Aquin, Ferron, Antonine Maillet et VictorLévy Beaulieu, il notait: «Le récit de notre temps ne peut être qu'inache- 
vé ${ }^{25}$. Ce jugement n'est pas qu'une belle conclusion d'article; il vaut pour l'ensemble du texte romanesque et du texte poétique de notre époque; il vaut également pour le propre discours du critique. Une œuvre s'élabore, une pensée se forme, comme "une littérature se fait». G. Marcotte est toujours attentif à ce qui prend lentement forme, autant dans une œuvre particulière que dans une époque donnée.

\section{La mise en ceuvres}

II serait trop long de prendre chaque œuvre chronologiquement pour montrer comment cette attention passionnée se manifeste et comment s'affinent et s'affermissent les instruments d'analyse. Arrêtons-nous-y tout de même un instant. Une Littérature qui se fait (1962) se voulait témoignage et acte de foi. G. Marcotte y parle d'œuvres «avec lesquelles je veux engager dès maintenant le dialogue "26. L'édition de 1968 ajoute, aux études de romanciers et de poètes, le texte capital: "L'expérience du vertige dans le roman canadien-français ", d'où j'extrais seulement ceci, pour montrer que G. M. a parsemé son œuvre critique - la preuve en serait facile à faire - de jugements verdictifs que personne ne peut ignorer:

Les œuvres les plus férocement critiques du roman français - je pense à Sartre, à Camus, à Céline - ne démolissent qu'en fonction d'un idéal de reconstruction, d'un ensemble de valeurs qui pourraient fonder une vie nouvelle. Desvaleurs existent: celles qui sont combattues, et celles qui sont proposées. Dans le roman canadien-français, par contre, on touche aussitôt le fond, et l'on y reste. Rien ne résiste au désespoir, et rien n'en procède. Des valeurs passent, ou plus justement des images de valeurs, mais si rapidement qu'on ne réussit à en retenir aucune. Les thèmes traités par les grandes littératures européennes, on les retrouve ici, car nos romanciers ont lu, et réfléchi; mais ils sont traités, projetés de telle façon qu'ils ne forment plus qu'une vertigineuse, une étourdissante litanie d'absence ${ }^{27}$.

Quant au projet de Présence de la critique (1966), il consiste à réunir d'importants textes de notre critique et à valoriser cette dernière. Le critère adopté est que les textes soient "en commerce direct, immédiat, avec les cuvres "28. Il faut situer dans son époque un discours qui parlant de l'essayiste le fait reconnaître «à la personnalité de sa démarche, à l'originalité de son langage, qui font de ses écrits de véritables créations". De nos jours, ces qualités sont tellement vues comme une présupposition qu'on n'en parle même plus, sauf en cas d'extrême embêtement. Mais qu'importe, ce livre bien conçu mettait notre critique sur la carte de la littérature du Québec. L'œuvre qui suit est le Temps des poètes (1969): elle marque un tournant dans sa pratique de la critique. "Le temps est venu, me semble-til, de dépasser ces considérations de psychologie personnelle et collective. Elles ont leur poids, leur sens, mais elles ont le tort de faire abstraction de l'œuvre en ce qu'elle a de plus spécifique: la forme même du langage” 29 . Ainsi donc c'est à partir de cette cuvre que G. Marcotte passe du 
thème psychologique et moral à la fonction propre de la poésie, à son "organisation intime", et à "la mise en question indéfinie du sens donné". Le sous-titre: “description critique» ne rend pas justice à cet important changement de cap. Pour une fois, la modestie aura joué un tour à son auteur, car c'est d'une phénoménologie des textes qu'il s'agit; mais allez donc mettre, ne serait-ce qu'en sous-titre, un pareil écriteau.

Pour ce qui est des Bonnes rencontres (1971), ce sont vraiment des chroniques littéraires, sans prétention, quarante et une si j'ai bien compté. Dans l'ensemble de l'œuvre, on pourrait les situer comme "l'artisanat préalable», ou la somme des lectures disparates que tout individu fait au cours de sa vie. C'est sur le Roman à l'imparfait (1976) qu'il faudrait s'arrêter longuement. On y verrait que l'idée-force a trait à la mimesis: "De Trente Arpents à Une Saison dans la vie d'Emmanuel, c'est tout le roman, le système même de la représentation, qui change " ${ }^{30}$. Les formules heureuses abondent: "Comment réciter ce monde nouveau que nous faisons et qui nous fait ", formulation que ne répudierait ni le sémioticien ni le sociologue. D'autres expressions: "le roman comme im-parfait, roman de l'imperfection, de l'inachèvement, de ce qui se donne, dans son projet même, comme expérience de langage jamais terminée, interminable ", font penser autant à Blanchot qu'à Barthes. Mais soyons certains que nulle part dans ce livre on ne trouve ce mimétisme qui indique toujours la plus grande faiblesse de la critique. Comme toujours, G. Marcotte propose ses lectures propres, mais ici nulle indécision dans le choix des positions critiques, nul flottement dans l'affirmation des lieux théoriques d'où l'on parle, aucune faiblesse dans l'homogénéité de la composition ni dans le traitement de quatre discours romanesques.

Je terminerai par quelques mots sur deux articles qui contiennent sans doute les idées-forces du critique. Le premier: "Les problèmes du capitaine ${ }^{31}$. Parlant du malaise qui a saisi l'institution littéraire, de la "déprime" qui affecte la scène de notre littérature, G. Marcotte trouve l'explication suivante: “L'ensemble de l'institution littéraire a glissé hors de la configuration idéologique qui l'englobait au cours des années soixante". II ajoute: "Les poèmes, les romans ne reçoivent leur pleine légitimité que de leur appartenance à la littérature nationale. Celles qui regimbent, on les y fait entrer, de gré ou de force. II n'y a plus, constate le critique, de concordance entre le projet littéraire, le projet social et le projet national m. Notre champ littéraire s'est morcelé ; il n'est plus unitaire comme autrefois. II conclut par un autre de ces verdicts, coutumiers, mais contre lesquels on ne peut rien: "L'inquiétude sur notre destin littéraire est une des constantes les plus fermes, et les plus embêtantes, de notre littérature». Ne faut-il pas voir dans cette inquiétude un signe non trompeur qu'on est définitivement sorti de l'idéologie du pays? La problématique de la littérature nationale est devenue secondaire, mineure. N'est-ce pas un signe que notre pays possède sa littérature, à défaut que ce soit notre littérature qui ait son pays? 
Le second article s'intitule théâtralement: "Qui a peur du pygargue roux ? " 32 Un texte majeur de la critique québécoise, une réflexion sur l'écriture, sur la littérature, comme j'en connais peu ici. Évoquant Lautréamont - et son oiseau de papier qui vient tout droit de Hugo - Bataille, Blanchot, McLuhan, Gilles Marcotte accède ici à un sommet de l'interrogation que porte la littérature et qu'on lui pose constamment. La question centrale pourrait être la suivante: l'écriture deviendra-t-elle «simple critique d'elle-même, l'arme de sa disparition"? Nous ne sommes plus dans «la théorie littéraire nationale"; " nous sommes enfin dans la pauvreté, l'essentielle et féconde pauvreté de l'écriture». Quel commentaire apporter à cela? Aucun, sinon se taire. Qu'il me suffise de citer deux textes. L'un sur un personnage:

Dulcinée est la raison de l'œuvre, mais une raison que l'œuvre se donne, non pas une raison qu'elle reçoit d'en-haut, ou d'ailleurs.

L'autre sur un auteur:

La grandeur de Don Quichotte est de savoir et de ne pas savoir; de contrôler la lucidité par l'ignorance, et l'ignorance par la lucidité.

On sait tout sur la littérature quand on commence de n'en plus rien savoir. II reste quand même quelque chose à écrire. II reste à lire l'œuvre de Gilles Marcotte. Quelque chose commence quand tout s'achève.

P.S.-

Juste après avoir terminé cet article, j'ai. mis la main sur le dernier livre de Gilles Marcotte: la Littérature et le reste (livre de lettres) (Quinze/ prose exacte, 1980,185 p.). C'est un échange de lettres avec André Brochu. Je mettrais une sourdine à l'affirmation de G. M.: “Chez nous, au Québec, il n'y en a pas (de grands critiques); de bons critiques, oui, dans les journaux, les universités ou ailleurs, mais aucun qui ait produit une cuvre marquante, neuve, originale, capable d'entrer en concurrence et en dialogue avec ce qui se fabrique ailleurs" (p. 149). Et Brochu renchérit en contrepoint: “Vous aurez compris que je vous donne raison sur tous les points. y compris sur l'absence d'œuvre critique originale et forte au Québec» (p. 156). Dans les deux cas, c'est pousser l'humilité un peu loin, surtout avec la performance dont nous sommes témoins dans ce livre. Les temps forts de ce dialogue, portant sur la littérature, se ramènent à trois: a) une première partie où l'on voit aux prises le Dieu du chrétien et le Dieu du philosophe, par suppôt interposé; b) une analyse forte et poussée de la Nausée où brillent les démonstrations (structurales et narratives) de Brochu; c) un discours magnifique sur la critique où Brochu, ex-thématicien et néo-structuraliste, multiplie des réflexions justes- et souvent pessimisteset où Marcotte, vieux routier de la sociocritique, volontiers socratique, ne cache pas son bonheur d'être optimiste. Le livre se dévore en une jour- 
née. - Ce n'est pas le tout dernier propos de G. M. qui contredira l'idée et le fond même de mon article: «Écrire des lettres, n'est-ce pas consentir au non-fini? J'y ai pris bien du plaisir" (p. 185). Il avait dit précédemment: "La théorie n'est pas mon fort " (p. 178), ce dont on se doutait bien. Ce livre sérieux, supposant un travail colossal de réflexion et d'écriture, est plein de vie et de pointes. Les métaphores sportives et militaires m'ont beaucoup amusé, dans la première partie du livre. C'était mêlé à de la religion. Après que les correspondants eurent accroché leurs patins et «défroqué", ils sont apparus comme de simples mortels, au pas lent et à l'air ordinaire. Deux passionnés de littérature, dont on attend encore beaucoup.

1. G.M. à André Major, à propos du Temps des poètes, Le Devoir, 3 janvier 1970 , p. 9.

2. Les Bonnes rencontres, HMH, 1971, p. 10.

3. Le Roman à l'imparfait, La Presse, 1976, p. 187.

4. Idem.

5. Le Temps des poètes, HMH, 1969, p. 14.

6. Les Bonnes rencontres, p. 10.

7. Une Littérature qui se fait, HMH, 1962 ; éd. augmentée, 1968, p. 7.

8. Le Roman à l'imparfait, p. 19.

9. "Qui a peur du pygargue roux", Liberté, no 84, 1972, p. 101.

10. Le Temps des poètes, p. 17.

11. Ibid., p. 19.

12. "Les problèmes du capitaine", Liberté, no III, 1977, p. 79.

13. Ibid., p. 79 .

14. Ibid., p. 85.

15. G. M. à André Major, à propos du Temps des poètes, Le Devoir, 3 janvier 1970, p. 9.

16. Préface à J.-C. Falardeau, Imaginaire social et littérature, HMH, 1974, p. 12.

17. Jean Éthier-Blais, Le Devoir, 3 janvier 1970, p. 9.

18. Préface à J.-C. Falardeau, op. cit., p. 11.

19. Robert Vigneault, “La critique...", Études françaises, mai 1972, pp. 199-201.

20. Liberté, no 84, p. 102.

21. Revue de l'Université Laurentienne, février 1978, pp. 67-75.

22. Le Roman à l'imparfait, pp. 10-13.

23. Ibid., p. 18.

24. Jacques Michon, Les Lettres québécoises, no 6, avril-mai 1977, p. 23.

25. "La problématique du récit dans le roman québécois d'aujourd'hui ", Revue des Sciences humaines, Lille III, no 173, janvier-mars 1979, p. 68.

26. Une Littérature qui se fait, p. 7 .

27. Ibid., p. 65.

28. Présence de la critique, HMH, 1966, p. 13.

29. Le Temps des poètes, p. 13.

30. Le Roman à l'imparfait, p. 13. Je renvoie le lecteur aux comptes rendus de ce livre faits par Jacques Michon dans Lettres québécoises, avril-mai 1977, pp. 22, 23, 49 et par Jean Fisette dans Livres et Auteurs québécois 1976, pp. 229-233.

31. "Les problèmes du capitaine", Liberté, no III, mai-juin 1977, pp. 78-86.

32. "Qui a peur du pygargue roux?", Liberté, no 84, 1972, pp. 94-115. 